\title{
PULSATION IN RAPIDLY ROTATING STARS
}

\author{
MAURICE J. CLEMENT \\ Department of Astronomy, University of Toronto, Toronto, ON, Canada M5S1A7
}

\begin{abstract}
The line-profile variables observed on the upper main sequence have been interpreted by some astronomers to be the manifestation of nonaxisymmetric oscillations. More specifically, most of these variables can be modelled by prograde or corotating equatorial waves. In the absence of rotation, these waves have surface velocity distributions which are given simply by spherical harmonics. Unfortunately, the corresponding velocity fields in the presence of rotation are much more difficult to calculate. In this paper, I will summarize what is known about the effect of rapid rotation on the normal mode eigenfunctions of main sequence stars. The principal conclusions are as follows: Low-order, axisymmetric modes couple very strongly to rotation and their velocity distributions are very much different from those of their zero-rotation counterparts. On the other hand, higher-order (shorter wavelength), nonaxisymmetric modes couple only weakly to rotation and, therefore, retain many of the spherical harmonic properties that they possess in the absence of rotation.
\end{abstract}

\section{Stellar Normal Modes: A Review}

\subsection{The Discrete Spectrum of Nonradial Modes}

The discrete modes are a measure of the global stability of the system. For spherical symmetry, the eigenfunctions are separable in the coordinates and their angular dependence is given by spherical harmonics:

$$
f_{k}(r) P_{\ell}^{m}(\cos \theta) e^{i m \varphi}\left\{\begin{aligned}
k & : \text { radial order } \\
\ell & : \text { polar order } \\
m: & \text { azimuthal order }
\end{aligned}\right.
$$

In this case, the eigenfrequencies are degenerate in $m$. Rotation, however, lifts the degeneracy and each mode involves a mixture of spherical harmonics. In general, the discrete modes fit into the following classification scheme:

1. Fundamental (f) modes:

These are analogs of the divergence-free Kelvin modes of an incompressible, uniform density sphere. They occur only in the lowest radial order which corresponds normally to an absence of radial nodes in the eigenfunction although there are exceptions in the limit of very high mass concentrations.

2. Pressure (p) modes:

Pressure is the main restoring force in this case. The modes are longitudinal (acoustic) waves whose motion is primarily radial and large only near the surface. The pressure variations are relatively large. 
3. Gravity (g) modes:

Gravity is the main restoring force; the motion is more horizontal than for the $p$-modes and can be large even in the deep interior. The pressure variations are small.

4. Toroidal ( $t$ ) modes:

The inertial forces of rotation are the restoring agents for these waves. The motion is mainly horizontal with very small variations in pressure and density.

The $p$-and $g$-modes form two separate spectra with $k$ running from 0 to $\infty$ for a given $\ell$ and $m$. In the first case, the eigenfrequency increases with $k$ whereas, in the second case, it decreases.

\subsection{The Continuous Spectrum of Nonradial Modes}

Stars can have branches of continuous modes; i.e., in a particular range of frequency, every value is an eigenvalue of the system. Thus, the modes are not discrete. (Computationally, they become strictly continuous only in the limit of zero grid spacing.)

Continuous modes appear in other areas of physics (e.g., geophysics). In astronomy, they were first noticed by Aizenman \& Perdang (1973) in some secular stability studies of main sequence stars. The associated eigenfunctions have a nonanalytic behavior along 2D-surfaces in the interiors of the models.

Perdang $(1976,1977)$ has shown that the stellar stability equations generally allow a class of continuous modes if one drops the "smooth and continuous" restriction. Sometimes, one can transform variables in such a way as to expose singularities in the equations and these give rise to nonanalytic solutions, not all of which are necessarily physical. That is, continuous modes may be introduced artificially into a problem by a poor choice of variables. For example, Clement (1981) encountered nonphysical continuous modes as a result of reducing the number of independent variables to make the problem more numerically tractable.

Continuous modes which are real are believed to be a measure of the local stability of the equilibrium state. Indeed, Perdang has shown that there is a branch of continuous modes associated with convection zones in stars.

\section{Methods for Computing the Normal Modes}

At one time or another, I have tested or employed all of the following techniques but in this paper only the results of methods 2 and 3 are summarized.

1. Collocation and Least Squares Fitting:

Trial functions are fitted to the partial differential equations. The idea is conceptually simple but the solutions are crude and they don't always 
converge with an increasing number of coefficients. Also, spurious nonphysical solutions commonly appear.

2. Variational/Tensor Virial Methods:

For analytic trial functions, all solutions are real physical solutions of the equations of motion and all discrete modes appear with no continuous branches. But the solutions are not exact - they are only the best ones for the chosen basis functions. Furthermore, the "ideal" set of basis functions that should be used for rotating configurations is unknown at the present time.

3. Exact 2D/3D Solutions of the Equations of Motion:

These are exact solutions but, unfortunately, the method permits both continuous and discrete modes and the former, if present, can hide the latter.

4. Hydrodynamical Simulations of Oscillating, Rotating Stars:

In principle, one can include convection, turbulence, and nonadiabatic effects in a natural way, but the method is computationally intensive and certainly overkill if only the eigenfunctions are required.

\section{Axisymmetric Modes}

\subsection{Direct Integration of the Equations of Motion}

For a first look at the axisymmetric modes, I solved the difference equations directly on a two-dimensional grid. Refer to Clement (1981) for the details. But, in summary, solutions of the form

$$
\xi(\mathbf{r}, t)=\xi(r, \theta) e^{i \omega t}
$$

were found numerically for $15 M_{\odot}$ main sequence models as well as for some polytropes to test the effects of different density gradients. The independent variables at each mesh point were (i) $\xi_{n}$ (the normal component of $\xi$ ), (ii) $\nabla \cdot \xi$, and (iii) $\delta \phi$ (the potential perturbation). The elimination of two of the displacement components reduces the matrix inversion required at each mesh point to order 3 and greatly speeds up the calculation. The eigenfrequency $\omega$ follows from the requirement that the determinant of the $3 \times 3$ matrix vanish at the equator.

Unfortunately, this choice of variables introduces singularities into the equations and results in the appearance of continuous modes. If this calculation were to be repeated today with faster computers, it would probably be advisable to avoid the continuous modes by choosing a more straightforward set of independent variables.

\subsection{Results for Four Low-Order Modes}

Rotation has a strong effect on the spatial distribution of the eigenfunction amplitude. Fig. 1 illustrates two modes which are purely radial in the absence 


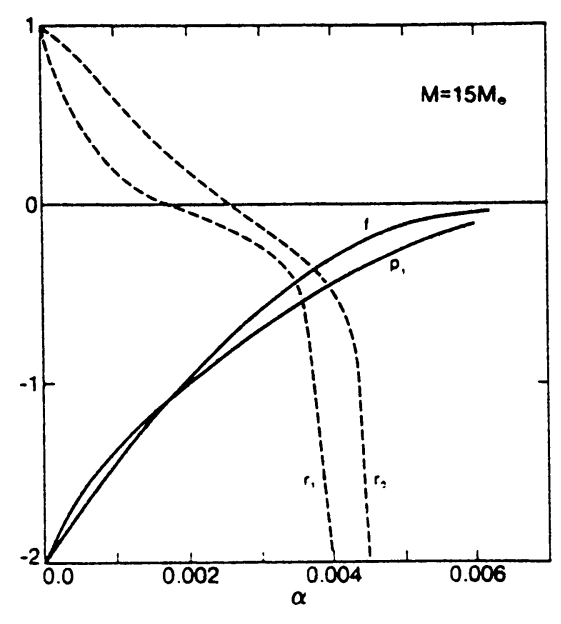

FiG. $1 a$

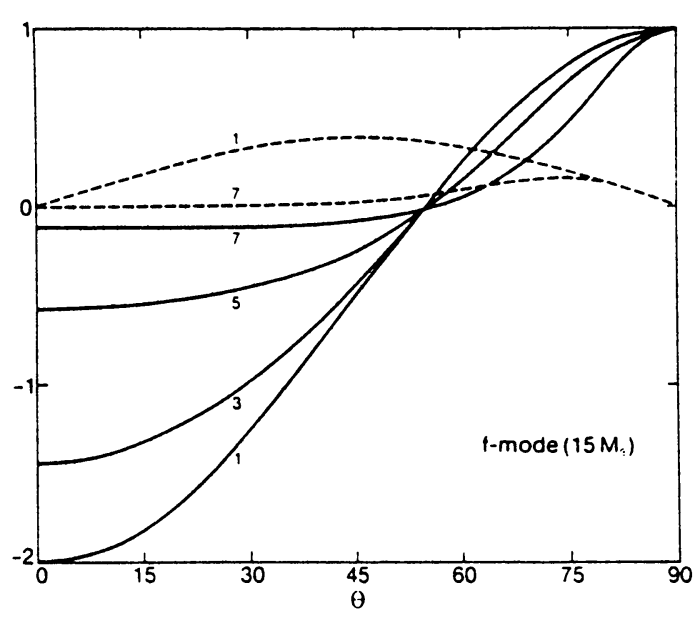

Fig. $1 b$

Fig. 1. (a) The effect of rigid rotation on the normalized eigenfunction amplitudes at either the pole or the equator of an $M=15 M_{\odot}$ main sequence model. Dashed curves, the normal or vertical component of the displacement, $\xi_{n}$, at the equator relative to $\xi_{n}$ at the pole for the $r_{0}$ and $r_{1}$ normal modes; solid curves, $\xi_{n}$ at the pole relative to $\xi_{n}$ at the equator for the $f$ and $p_{1}$ modes. The rotation parameter $\alpha$ is equal to $\Omega^{2} / 8 \pi G$. (b) The surface distributions of the horizontal and vertical displacements of the $M=15 M_{\odot}$ $f$-mode. Dashed curves, $\xi_{h}$ as a function of polar angle; solid curves, $\xi_{n}$ as a function of polar angle. The model numbers attached to each curve vary from $1(\alpha=0)$ to 7 $\left(\alpha=0.005\right.$ or $\left.v_{e q}=378 \mathrm{~km} \mathrm{~s}^{-1}\right)$. All the displacements are normalized with respect to the equatorial $\xi_{n}$.

of rotation: the lowest-order radial mode $r_{0}$ and its first overtone $r_{1}$. They both end up in the limit of rapid rotation with most of their amplitude confined to the equatorial zones. This same transformation with rotation is experienced by two initially $\ell=2$ modes $\left(f\right.$ and $\left.p_{1}\right)$. These results are confirmed by completely independent variational calculations applied to the case $m=0$. The variational method is described briefly in the next section.

\section{Nonaxisymmetric Modes}

\subsection{The Variational Method}

As applied to rapidly rotating stellar models, this approach and some results for upper main sequence stellar models can be found in Clement (1981, 1984, 1986, 1989). The method involves three basic steps:

1. Linearize the equations of motion by setting $\delta \mathbf{r} \equiv \xi(\mathbf{r}, t)=\xi(\mathbf{r}) e^{i \omega t}$ :

$$
-\omega^{2} \mathbf{A}(\xi)+\omega \mathbf{B}(\xi)+\mathbf{C}(\xi)=0 .
$$


2. Multiply by the complex conjugate $\xi^{*}$ and integrate over the equilibrium volume to obtain

$$
-\omega^{2} a+\omega b+c=0 \text {. }
$$

The (Hermitian) symmetry properties of the operators $\mathbf{A}, \mathbf{B}$, and $\mathbf{C}$ make this a variational equation. That is, $\omega$ is stationary for arbitrary variations of an eigensolution $\boldsymbol{\xi}$ (and conversely).

3. Choose a trial function involving linear combinations of "basis" functions or vectors $\mathbf{f}_{i}$ :

$$
\boldsymbol{\xi}=\sum_{i=1}^{N} c_{i} \mathbf{f}_{i}(\mathbf{r}) \text {. }
$$

The coefficients $c_{i}$ can be determined variationally by requiring

$$
\partial \omega / \partial c_{i}=0 \quad(i=1, N) \text {. }
$$

The resulting $N$ linear homogeneous equations will have a solution only if the determinant of the coefficient matrix vanishes. This condition yields $N$ normal modes for each equilibrium model.

\subsection{A Set of Basis Vectors for Variational Calculations WITH RAPID Rotation}

Ideally, the basis vectors $f_{i}$ should form a complete set (preferably orthogonal), and they should span the vector space of all possible eigenfunctions. Obviously, for practical reasons, one wants a set which is capable of describing an eigenfunction with a linear combination of a finite (i.e., small) number of $f_{i}$.

Inspired by Sabouti (1977a, 1977b, 1981), I tested a number of possibilities and finally chose a combination of three basis vector families: $p$ type, $g$-type, and $t$-type. They are approximately orthonormal and describe respectively $p$-modes, $g$-modes, and $t$-modes in the limit of small rotation (Clement 1989). With large rotation, however, the modes lose some of their unique character and become less distinguishable from one another. That is, the normal modes under the influence of rapid rotation can be represented accurately only by a mixture of $p$-type, $g$-type, and $t$-type basis vectors.

Each basis type is derivable from a scalar potential as follows:

1. $p$-type: $\rho \xi_{p}=\rho \nabla \phi_{1}$

2. $g$-type: $\quad \rho \boldsymbol{\xi}_{g}=\nabla \times\left(\nabla \times \phi_{2} \mathbf{g}\right)+A \mathbf{g} \times\left(\nabla \times \phi_{2} \mathbf{g}\right)$

3. $t$-type: $\rho \xi_{t}=\nabla \times \phi_{3} 8$

where $A \equiv(d \rho / d P)_{a d}-(d \rho / d P)$ measures the departure from adiabaticity and $\mathbf{g}$ is the local gravity. The $p$-type vectors generate acoustic, shear-free waves, the $g$-type are associated with isobaric waves, and the $t$-type yield constant density, horizontal waves. The three scalar potentials are given by

$$
\phi_{1}(\mathbf{r})=e^{i m \varphi} \sum_{k=m}^{K} \sum_{\ell=m}^{k} a_{k \ell} r^{k} P_{\ell}^{m}(\cos \theta),
$$




$$
\begin{aligned}
& \phi_{2}(\mathbf{r})=e^{i m \varphi} P(\mathbf{r}) \sum_{k=m}^{K} \sum_{\ell=m}^{k} b_{k \ell} r^{k} P_{\ell}^{m}(\cos \theta), \\
& \phi_{3}(\mathbf{r})=i e^{i m \varphi} \rho(\mathbf{r}) \sum_{k=m}^{K} \sum_{\ell=m}^{k} c_{k \ell} r^{k-1} P_{\ell+1}^{m}(\cos \theta) .
\end{aligned}
$$

Notice the presence of either the pressure or the density in two of the potentials. This ensures the correct behavior at a model's outer boundary. Also, note that the toroidal potential is imaginary and involves odd Legendre polynomials for even modes. In practice, the parameter $K$ was chosen large enough to yield 8 or 9 radial orders and about 150 normal modes for each equilibrium model.

\subsection{Mixing of the Basis Vector Types}

For slow rotation, a basis vector family describes more or less accurately its corresponding normal mode type. For example, the $p$-modes involve primarily $p$-type basis vectors as shown in Table $I$ by the large value of $p_{\text {mix }}$ (the fraction of the total amplitude due to $p$-type basis vectors). However, with rapid rotation, the basis types mix and, in particular, the motions of all the modes becomes more toroidal in their nature as indicated by the value of $t_{\text {mix }}=1-p_{\text {mix }}-g_{\text {mix }}$. On the other hand, in the absence of rotation, $t_{\text {mix }}$ is zero because the toroidal vectors uncouple from the other basis types in that limit.

\section{TABLE I}

\begin{tabular}{|c|c|c|c|c|c|c|}
\hline \multirow[b]{2}{*}{ Mode } & \multirow[b]{2}{*}{ factor } & \multicolumn{5}{|c|}{$\Omega /\left(2 \pi G \rho_{c}\right)^{1 / 2}$} \\
\hline & & 0.00 & 0.02 & 0.04 & 0.06 & 0.08 \\
\hline \multirow[t]{2}{*}{$p(1,4,4)$} & $P_{\text {mix }}$ & 0.92 & 0.93 & 0.90 & 0.72 & 0.58 \\
\hline & $\mathbf{g}_{\operatorname{mix}}$ & 0.08 & 0.05 & 0.01 & 0.08 & 0.07 \\
\hline \multirow[t]{2}{*}{$f(0,4,4)$} & $\mathbf{P}_{\text {mix }}$ & 0.74 & 0.73 & 0.73 & 0.46 & 0.43 \\
\hline & $g_{\text {mix }}$ & 0.26 & 0.21 & 0.15 & 0.15 & 0.12 \\
\hline \multirow[t]{2}{*}{$g(1,4,4)$} & $P_{\operatorname{mix}}$ & 0.36 & 0.28 & 0.20 & 0.12 & 0.11 \\
\hline & $\mathbf{g}_{\operatorname{mix}}$ & 0.64 & 0.59 & 0.54 & 0.48 & 0.40 \\
\hline
\end{tabular}

The $P_{\text {mix }}$ and $g_{\text {mix }}$ Factors for Some $(\ell=m=4)$-Modes 


\subsection{Effect of Rapid Rotation on Nonaxisymmetric Eigenfunc- TIONS}

Unlike the axisymmetric case, the rotational coupling of modes to adjacent spherical harmonics is not particularly strong. Aside from the motion becoming more toroidal as mentioned in the preceding section, the modes maintain their basic zero-rotation spatial distribution. There is some focusing of the amplitude into the equatorial regions as illustrated in Fig. 2, but the change is not so dramatic as in the $m=0$ case because the polar amplitude is always zero for the nonaxisymmetric modes regardless of the rotation rate. Refer to Clement (1989) for more details. I should emphasize that this conclusion applies only to the lowest half dozen radial orders that $I$ have been able to compute. The effect of rotation on very high-order modes remains unknown.
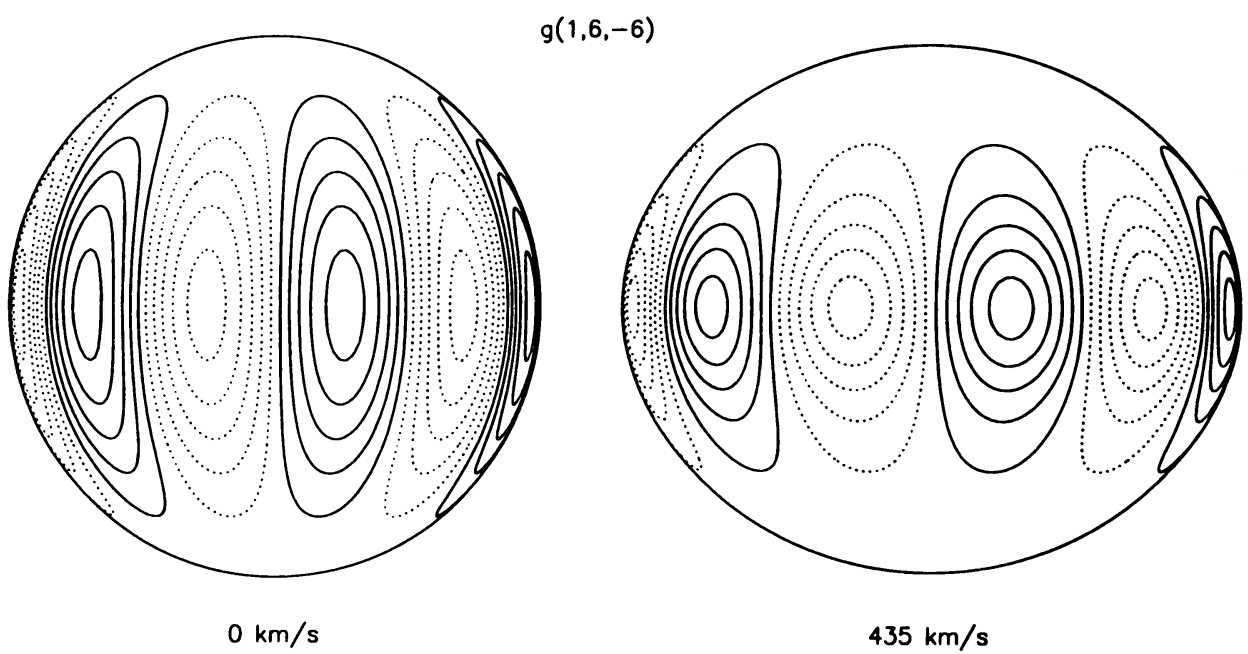

Fig. 2. The azimuthal velocity component of the $\ell=6$ prograde sectorial $g$-mode for zero rotation on the left and rapid rotation $\left(v_{e q}=435 \mathrm{~km} \mathrm{~s}^{-1}\right)$ on the right. The solid contours indicate positive velocity and the dashed curves, negative velocity. There is a significant focusing of amplitude toward the equatorial zones by rotation.

\subsection{Line Profile Variations}

The prograde sectorial $g$-modes appear to be the most likely source of the line profile variations observed in various B-stars on the main sequence. Some of the periods are quite long, indicating perhaps the presence of high radial orders. However, one must be careful here because rotation destabilizes the retrograde $g$-modes (Clement 1989), especially the $\ell=m$ sectorial modes. Thus, the eigenfrequency $\omega$ can pass through zero, making the modes prograde with very long periods if the rotation rate is just above the neutral 
stability point. Here, the modes become secularly unstable and their amplitudes may grow if there is a significant dissipation of energy (Friedman \& Schutz 1978).

As an example, Fig. 3 illustrates the effects of rotation on the line profile variations due to the lowest radial order $\ell=6$ sectorial $g$-modes. These results are representative of orders up to $k=6$, say. Again, I can say nothing about the very high radial orders that appear to be present in some variables, but note my remarks in the preceding paragraph. It should also be pointed out that these line profiles are highly schematic or ideal in the sense that they completely ignore atmospheric effects such as shocks which may give profiles a sharper, less coherent appearance.

Doppler Profile - $g(1,6,6)$

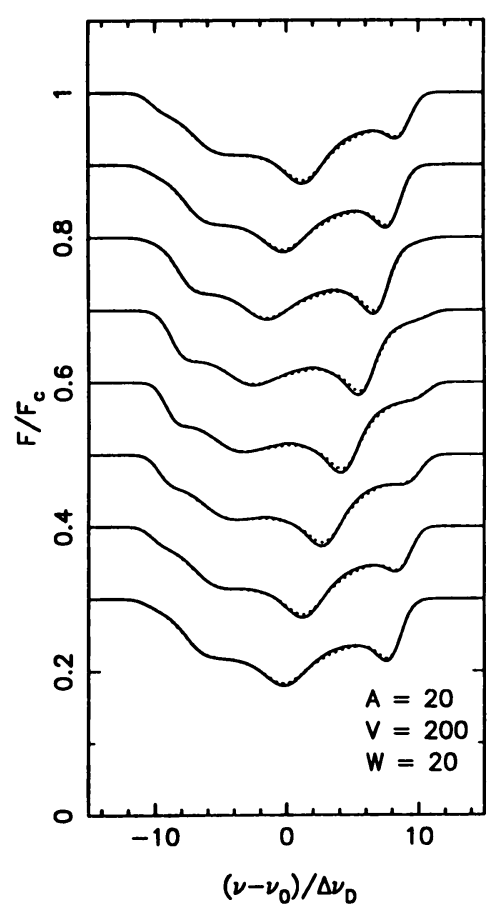

Doppler Profile - $g(1,6,-6)$

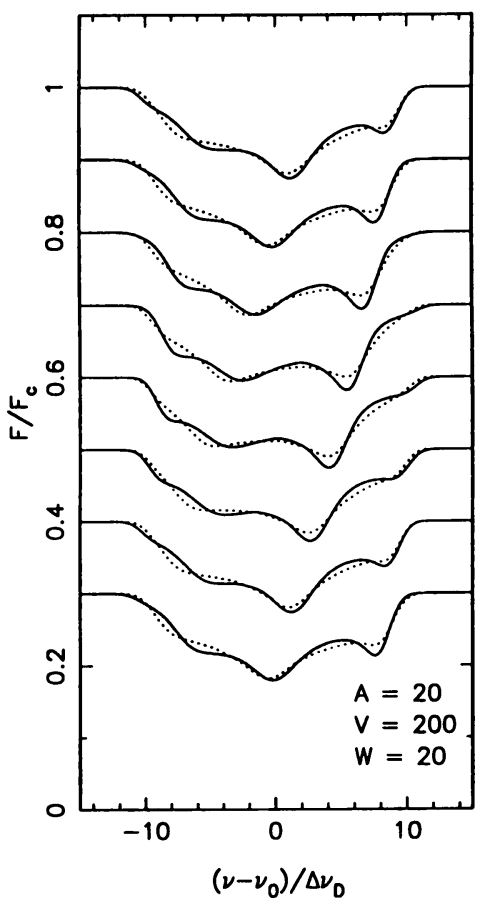

Fig. 3. Line profile variations due to the $\ell=6$ sectorial $g$-modes in a $15 M_{\odot}$ model $(m=6$ on the left and $m=-6$ on the right). All the profiles are normalized in the same way; that is, they are broadened with a rotational velocity of $200 \mathrm{~km} \mathrm{~s}^{-1}$ and incorporate an intrinsic broadening in the Voigt function of $20 \mathrm{~km} \mathrm{~s}^{-1}$. The solid curves were computed with the zero rotation eigenfunctions (which are pure spherical harmonics) whereas the dotted curves were found using the corresponding eigenfunction for a rapidly rotating model $\left(v_{e q}=435 \mathrm{~km} \mathrm{~s}^{-1}\right)$.

That said, observe that even relatively fast rotation (namely, $v_{e q}=435$ $\mathrm{km} \mathrm{s}^{-1}$ ), changes the profiles in only a modest way although the $m=-6$ 
mode does give rise to larger effects than the $m=6$ one. Note that both the rotating modes are prograde with $\Omega / \omega$ equal to $0.095(m=-6)$ and 0.66 $(m=6)$. Thus, the mode which is retrograde at small rotation appears as a high-order, prograde mode at a somewhat faster rotation. One can push the 0.66 ratio up to any higher value just by choosing an appropriate slower rotation rate. [In the figure, the $m=6$ solid-line profiles indicate bumps moving in the prograde direction, opposite to the correct sense; this was done only to make the comparison with the rotating eigenfunction easier.]

\section{An Alternative Approach: Hydrodynamical Simulations}

As part of a long term study of the interior dynamics of rotating stars, I am now tackling this problem with a 2D/3D hydrodynamics code. Conserving energy and modeling the subgrid-scale viscosity were major problems which have now been solved (Clement 1993). This approach can simulate convection, turbulence, pulsation, and rotation simultaneously. Fourier analyses of the velocity time series can be used to identify pressure, gravity, and Kelvin modes and to follow the evolution of the oscillation spectrum. For example, starting from arbitrary initial conditions, one can observe long period gravity modes quickly die out in the stellar core. Thus, it should be possible with this technique to extract the normal modes and their dependence on rotation.

\section{Conclusions}

Low-order, axisymmetric modes are strongly affected by rotation. In the limit of high angular velocity, the oscillation amplitude is confined to the equatorial regions. On the other hand, nonaxisymmetric modes and, in particular, the sectorial $(\ell=|m|)$ modes do not "see" the rotational distortion of the star and retain many of their zero-rotation characteristics. On closer examination, the oscillation amplitude is focused more into the equatorial zones and the motion becomes more toroidal in its nature as shown by the larger mixing ratio for the toroidal basis vectors. The weak coupling of the nonaxisymmetric modes to adjacent harmonics in the presence of rigid rotation will probably carry over to differential rotation as well except perhaps in the extreme case where the angular velocity scale length becomes comparable to the pulsation wavelength.

\section{Acknowledgements}

This work has been supported in part by the Natural Sciences and Engineering Research Council of Canada and by the University of Toronto. 


\section{References}

Aerts, C. \& Waelkens, C.: 1993, Astron. Astrophys. 273, 135.

Aizenman, M. \& Perdang, J.: 1973, Astron. Astrophys. 23, 209.

Clement, M. J.: 1981, Astrophys. J. 249, 746.

Clement, M. J.: 1984, Astrophys. J. 276, 724.

Clement, M. J.: 1986, Astrophys. J. 301, 185.

Clement, M. J.: 1989, Astrophys. J. 339, 1022.

Clement, M. J.: 1993, Astrophys. J. 406, 651.

Friedman, J. L. \& Schutz, B. F.: 1978, Astrophys. J. $222,281$.

Perdang, J.: 1976, Astrophys. Space Sci. 44, 177.

Perdang, J.: 1977, Astrophys. Space Sci. 52, 313.

Sabouti, Y.: 1977a, Astron. Astrophys. 55, 327.

Sabouti, Y.: 1977b, Astron. Astrophys. Suppl. 28, 463.

Sabouti, Y.: 1981, Astron. Astrophys. 100, 319.

\section{Discussion}

Balona: You show the rotational velocity in $\mathrm{km} \mathrm{s}^{-1}$, but fail to specify the pulsation period of your modes. The case which is of greatest interest is the one for which the ratio of rotation to pulsation frequency is no longer small; i.e., when it is of order unity, for example. Do your calculations include this domain?

Clement: My variational calculations cover radial orders up to 8. Of these, the sectorial $g$-modes with $m=-\ell$ (i.e., the ones which are always prograde) fall in the range $\Omega / \omega<0.20$. However, the $m=\ell$ modes cover the whole $\Omega / \omega$ domain because, for them, $\omega$ passes through zero at some rotation rate whereupon they become prograde with arbitrarily long periods. Refer to Clement (1989) for plots of eigenfrequency versus rotation rate.

Owocki: I may have missed a simple point, but could you give the critical angular velocity for the equilibrium models that you use. That is, what fraction of critical rotation are your assumed rates? Also, do you include oblateness and gravity darkening effects in your line profile calculations?

Clement: For the $15 M_{\odot}$ models which I was using, the critical equatorial velocity is around $600 \mathrm{~km} \mathrm{~s}^{-1}$. The fastest rotating model which I showed in my talk has a velocity of $435 \mathrm{~km} \mathrm{~s}^{-1}$ corresponding to an equatorial gravity which is 0.43 of the polar value. I did include oblateness and limb darkening effects in my calculations but not those of gravity darkening. I might include the latter effects in the future although limb darkening leads to only small changes in the profile variations.

Dziembowski: What is the cause of the continuous normal mode spectrum in the case of rigid rotation?

Clement: This is a good question. Rigid rotation should not in principle introduce continuous modes although differential rotation can. I believe that 
the branches of continuous modes reported in my 1981 paper are nonphysical and the result of a poor choice of independent variables. I eliminated two of the velocity components in order to make the problem more numerically tractable and this introduced singularities into the equations.

Aerts: The effect of rotation depends completely on $\Omega / \omega$. For $\Omega / \omega<1$, we find that axisymmetric modes are hardly coupled (Aerts \& Waelkens 1993), while nonaxisymmetric modes are strongly coupled. That is, the effects of rotation on LPV's are a lot larger for nonaxisymmetric modes than for axisymmetric ones if $\Omega / \omega$ is small. So you should have given explicitly $\Omega / \omega$ for the modes which you discussed.

Clement: I have to disagree somewhat with what you say here. Your paper with Waelkens includes only rotational perturbations up to first order in $\Omega$. This excludes all second-order effects which I would argue are necessary for the axisymmetric modes since there are almost no first-order terms in that case. In particular, you do not include the rotational distortion of the equilibrium model. My numerical calculations are exact (i.e., they are not perturbation analyses) and were done with two completely independent methods: the variational approach and a direct solution of the equations of motion. Both techniques give the same result and indicate a strong rotational coupling for the axisymmetric modes as pointed out in my talk. For the nonaxisymmetric modes, on the other hand, the first-order terms are relevant and you are on safer ground. However, I believe that you have pushed your analysis too far. It is not correct to argue that if only $\Omega / \omega$ is less than unity then your results are valid. You must also check that the centrifugal force at the equator is much less than the gravitational force. Also, the change in the eigenvalue should be much less than the zero-rotation value. Neither of these additional restrictions is satisfied by many of your models. In particular, I believe that your profile calculations for $\Omega / \omega=0.5$ (and perhaps even for $\mathbf{0 . 2}$ for some modes) correspond to rotation rates close to or above the critical value. In such cases, you cannot ignore the oblateness of the model and other second-order effects. I think that if you restrict yourself to reasonable rates, consistent with a first-order perturbation analysis, you will find that the effect of rotation on the line profiles is not so large after all. Also, I recommend that you give up the practice of normalizing your profiles so that they have the same depth at a particular phase. This artificially enhances the effect of rotation as illustrated by the profiles in your paper which clearly have smaller and smaller equivalent widths with increasing rotation. 